\title{
Modeling and Control Design of Dynamic Voltage Restorer in Microgrids Based on a Novel Composite Controller
}

\author{
Yonghong Huang ${ }^{\dagger}$, Junjun Xu*, Yukun Sun* and Yuxiang Huang*
}

\begin{abstract}
A Dynamic Voltage Restorer (DVR) model is proposed to eliminate the short-term voltage disturbances that occur in the grid-connected mode, the switching between grid-connected mode and the stand-alone mode of a Microgrid. The proposed DVR structure is based on a conventional cascaded H-bridge multilevel inverter (MLI) topology; a novel composite control strategy is presented, which could ensure the compensation ability of voltage sag by the DVR. Moreover, the compensation to specified order of harmonic is added to implement effects that zerosteady error compensation to harmonic voltage in specified order of the presented control strategy; utilizing wind turbines-batteries units as DC energy storage components in the Microgrid, the operation cost of the DVR is reduced. When the Microgrid operates under stand-alone mode, the DVR can operate on microsource mode, which could ease the power supply from the main grid (distribution network) and consequently be favorable for energy saving and emission reduction. Simulation results validate the robustness and effective of the proposed DVR system.
\end{abstract}

Keywords: Dynamic voltage restorer, Microgrid, Short-term voltage disturbance, Composite control strategy, Compensation to harmonic voltage in specified order

\section{Introduction}

Although the Microgrid has numerous technical and economical advantages, it is usually located in tail of the main grid, which is very vulnerable to various disturbances of power quality [1-2]. Microsources (e.g. wind power, solar photovoltaic cells and fuel cells, etc.) alone are insufficient in meeting users' requirements of output voltage and frequency, and require the help of power electronic technology in Microgrids. However, the use of power electronic equipments, to enhance the harmonic level, have been causing more or less negative effects on the power quality of critical loads.

The Microgrid is operated under two modes: (1) gridconnected and (2) stand-alone. When a switch between the two operation modes occur, there is a process of instantaneous voltage sag or swell, which negatively influences the microsources and power quality of critical loads in the Microgrid. In such, incalculable economic losses would be ensure if the appropriate control strategies and related equipments aren't employed. As it has been noticed, the negative drawbacks of the Microgrid could outweigh its' advantages [3-5].

Dynamic Voltage Restorers (DVRs) are effective equipments commonly used to regulate power quality disturbances [6,7]. In the past decade, a large number of research has focused on how to make DVR work more

$\dagger$ Corresponding Author: School of Electrical and Information Engineering, Jiangsu University, China. (hyh@ ujs.edu.cn)

* School of Electrical and Information Engineering, Jiangsu University,

China. (simulinkyer@163.com, syk@ujs.edu.cn, lxjsrn@126.com)

Received: October 5, 2015; Accepted: June 8, 2016 efficiently and at a lower operation cost, mainly aimed at DC energy storage $[8,9]$, the topological structure [10-12] and the compensation schemes or control strategies of DVR [13-17], etc. However, the bulk of current literature on DVR has focused chiefly on the main grid, and there has not been a similar extensive body of literature on the analysis and synthesis of DVR in Microgrids. With the further popularization of Microgrids, it is of significance to speed up the study work of DVR in Microgrids, maximize the role of Microgrids, weaken its' negative effects on critical loads and reduce economic losses.

In this paper, a suitable model of DVR in the Microgrid is proposed to address power quality disturbances caused during the two operation modes. (1) The proposed DVR system is based on a conventional cascaded H-bridge multilevel inverter (MLI) topology. (2) In the aspect of control strategy of MLI, a Sinusoidal Pulse Width Modulation (SPWM) based on composite control strategy composed of double closed-loop Proportional- Integral (PI) and Modified Proportional-Resonant (MPR) is presented to raise the operation performance of MLI and allow the DVR to further reduce the voltage harmonic characteristics of the system when compensating load voltage. Applying SPWM into the topology of MLI could obtain a higher equivalent switching frequency and lower switching losses. The inner closed-loop of current control with PI based on synchronous speed rotating coordinates could realize realtime tracking and compensation for load voltage, ensure that the load voltage is maintained at the rated value and improve the power quality and reliability (PQR). The outer closed-loop of voltage control with MPR, which has 
infinite open-loop gain at the point of resonant frequency, could theoretically achieve zero-steady error control and suppression to harmonic voltage in a specified order in the Microgrid, moreover, it could avoid the complex coordinate transformation, decoupling control and feedforward compensation control of conventional PR control and multiple-PI control, reduce the amount of calculation and simplify the complexity of the control system. (3) Utilizing wind turbines-batteries units as DC energy storage component in the Microgrid, would not only provide the required compensation energy handily but also avoid the use of additional energy storage devices of DVR. Such a design could simplify volume, reduce the operation and maintenance cost of the DVR. (4) When the Microgrid works under stand-alone mode, the DVR can operate in microsource mode, which could reduce the power supply from main grid and be favorable for energy saving and emission reduction. The theoretical analysis and simulation results based on Mat-lab/Simulink platform all validate the robustness and accuracy of the proposed DVR model.

\section{System Description}

The block diagram of the proposed DVR in the Microgri-d is shown in Fig. 1.

The proposed system consists of wind-turbines, $\mathrm{AC} / \mathrm{DC}$ converter, batteries, low set-up DC/DC converter, cascaded H-bridge MLI, control system for MLI, rectifier with Power Factor Correction (PFC) circuit and semiconductor switches $S_{1}, S_{2}, S_{3}$, circuit breaker (CB) and Point of Common Couple (PCC), the supply-side voltage $U_{S}$ and the loadside voltage $U_{L}$. In details, switch $\mathrm{CB}$ is a bidirectional one, which is used to control the DVR operation mode, when the $U_{S}$ is normal, CB is normally closed and DVR is under standby condition. On the contrary, when the $U_{S}$ fails, the CB is open and DVR is under working condition. Switch $S_{1}$ is used to control the operation of wind turbines. Switches $S_{2}$ and $S_{3}$ are used to control batteries charging. All the switches are operated by Energy Management System (EMS).

The proposed DVR system could operate in three modes in accordance with the Microgrid. Therefore, it could improve the utilization rate of equipments compared with the conventional ones in main grid. According to the changes of the measuring point voltage, through the status of system switches, it is clear to decide which mode the DVR operates in. The control logic of the DVR system operation modes is shown in Table 1 , where single " 1 " means open and single " 0 " means closed, voltage $U_{M G}$ is the Microgrid voltage.

\subsection{Grid-connected mode (compensation mode)}

In the grid-connected mode, the Microgrid remains connected to the main grid totally, and imports power from

Table 1. The control logic of the DVR system operation modes

\begin{tabular}{|c|c|c|c|c|}
\hline $\begin{array}{l}\text { Operation } \\
\text { Modes }\end{array}$ & $\begin{array}{c}\text { Measuring } \\
\text { point } \\
\text { Voltager }(\mathrm{V})\end{array}$ & Status of switches & $\begin{array}{l}\text { Wind } \\
\text { speed }\end{array}$ & $\begin{array}{l}\text { Status of } \\
\text { Batteries }\end{array}$ \\
\hline \multirow{2}{*}{$\begin{array}{c}\text { Compensation } \\
\text { Mode }\end{array}$} & $\begin{array}{l}\mathrm{U}_{\mathrm{S}}=380 \\
\mathrm{U}_{\mathrm{L}}=220\end{array}$ & 1101010 & - & Charging \\
\hline & $\begin{array}{l}\mathrm{U}_{\mathrm{S}}<380 \\
\mathrm{U}_{\mathrm{L}}<220\end{array}$ & 10110 & Stronger & Charging \\
\hline UPS n & $\begin{array}{c}\mathrm{U}_{\mathrm{MG}}<220 \\
\mathrm{U}_{\mathrm{L}}<220\end{array}$ & $1 \sim 00001$ & 一 & Dicharging \\
\hline \multirow{2}{*}{$\begin{array}{l}\text { Microsource } \\
\text { mode }\end{array}$} & $\begin{array}{c}\mathrm{U}_{\mathrm{S}}=0 \\
\mathrm{U}_{\mathrm{L}}=220\end{array}$ & $\begin{array}{llll}0 & 0 & 100\end{array}$ & Stronger & Charging \\
\hline & $\begin{array}{c}\mathrm{U}_{\mathrm{S}}=0 \\
\mathrm{U}_{\mathrm{L}}<220\end{array}$ & $\begin{array}{lllll}0 & 0 & 0 & 0 & 1\end{array}$ & Weaj & Dicharging \\
\hline
\end{tabular}

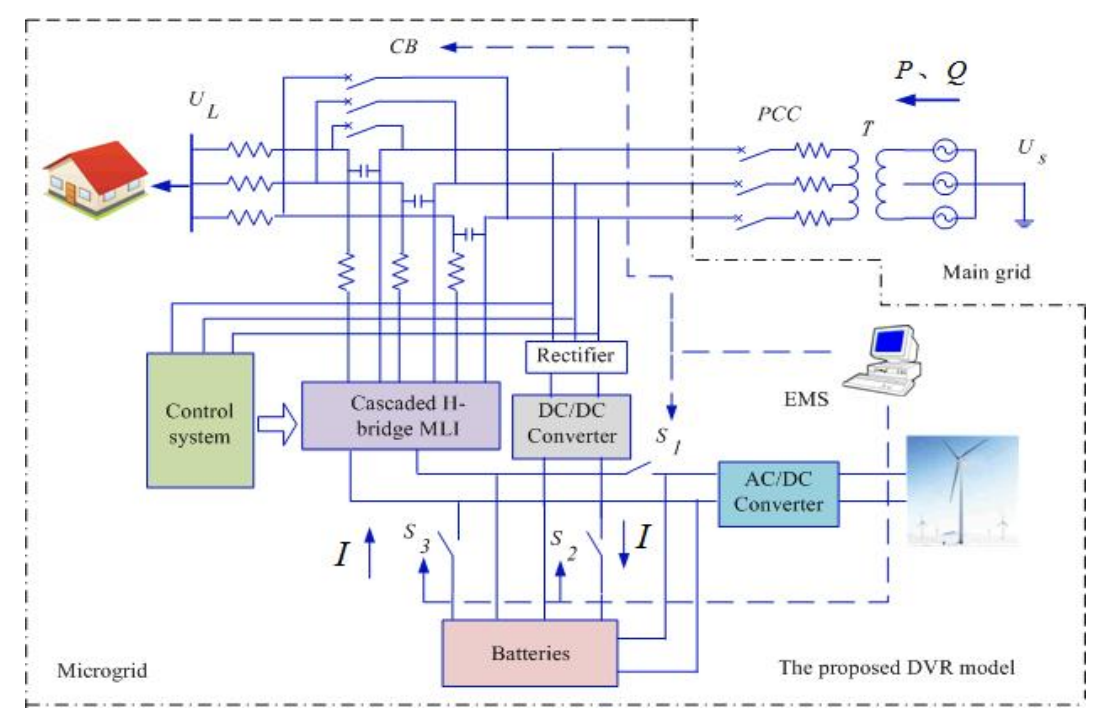

Fig. 1. The block diagram of the proposed DVR Model in a Microgrid 

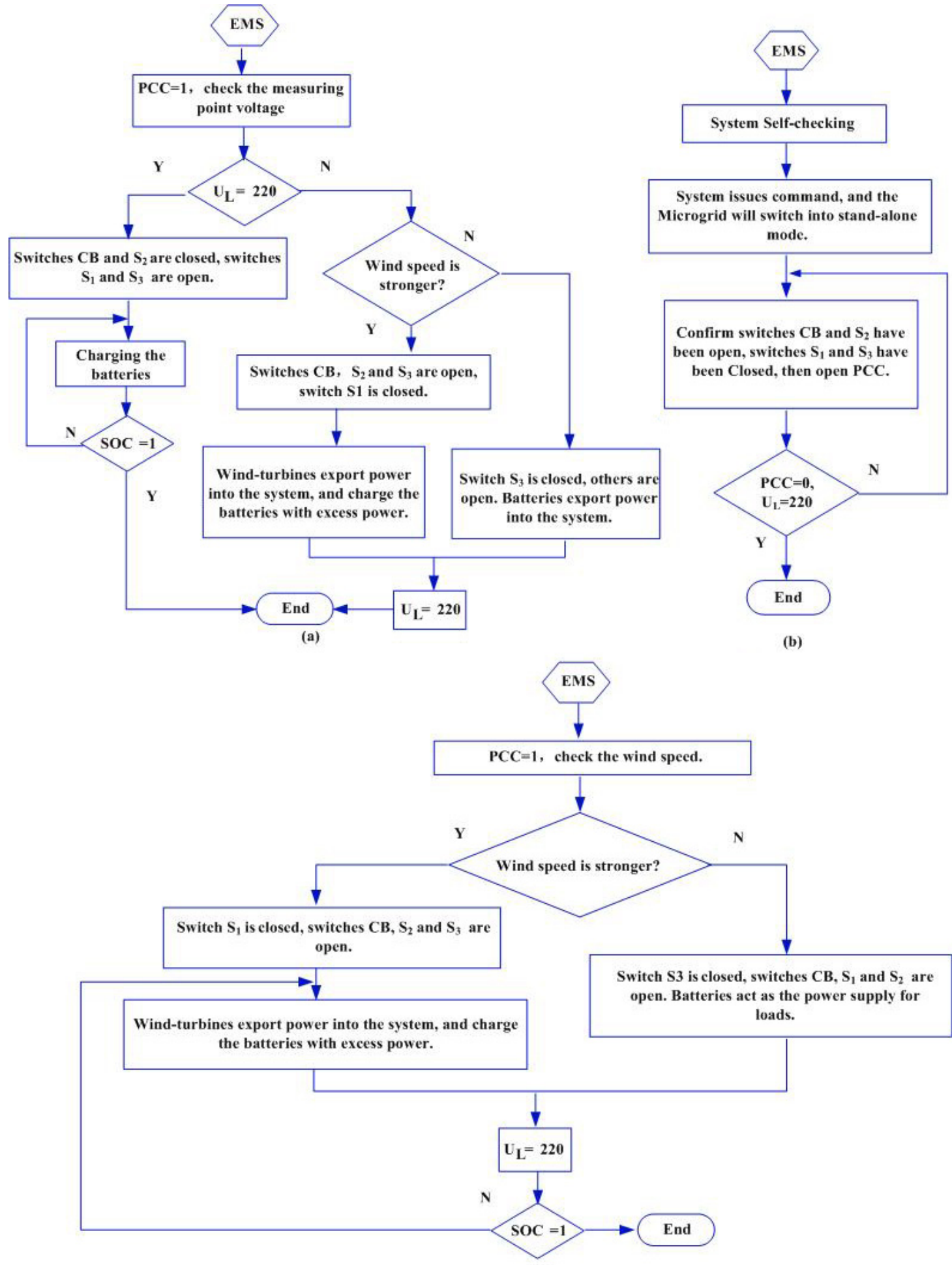

(c)

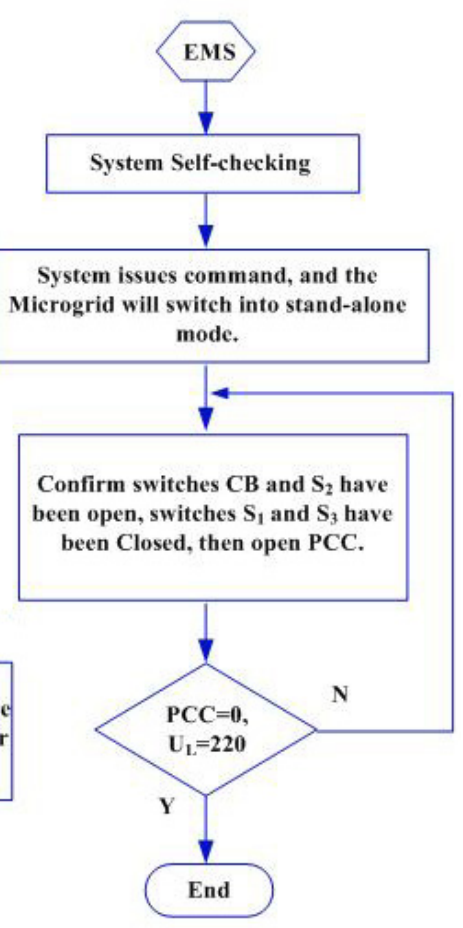

(b)

Fig. 2. The operational flowchart of EMS in all modes

the main grid to supply its critical loads, whereas switches $\mathrm{CB}$ and PCC are closed. When the voltage $U_{L}$ is normal, switches $S_{1}$ and $S_{3}$ are closed and $S_{2}$ is open, batteries could be charged by the rectifier and low set-up DC/DC converter, wind-turbines could also charge the batteries through the $\mathrm{AC} / \mathrm{DC}$ converter during appropriate weather conditions. In case of voltage sag in the main grid, switch CB should be open quickly and the DVR needs to operate in compensation mode so that it could ensure the PQR for priority loads in the Microgrid. The operational flowchart of EMS is shown in Fig. 2 (a).

Note that, the wind-turbines keep charging the batteries 


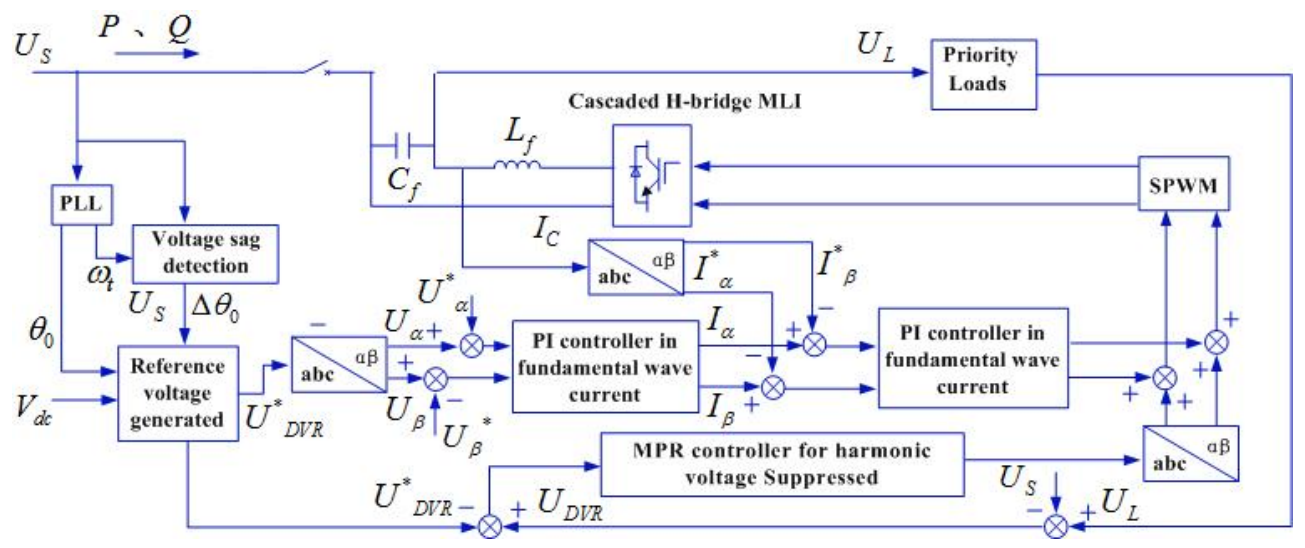

Fig. 3. The complete block diagram of proposed composite control strategy

whether the wind is strong or not, so as to optimize the State of Capacity (SOC).

\subsection{In the process of modes switching (UPS mode)}

When the Microgrid operates from grid-connected mode to stand-alone mode, instantaneous voltage disturbances would appear in the process of two modes switching and cause negative effects to the PQR of priority loads. In this mode, the proposed DVR could provide uninterrupted power supply for priority loads in order to ensure the Microgrid seamlessly switches between the two modes. When the Microgrid switches over to stand-alone mode, switch $S_{3}$ is open accordingly, so as to let batteries be in a discharge state. The operational flowchart of EMS in this mode is shown in Fig. 2 (b).

\subsection{Stand-alone mode (Microsouce mode)}

The Microgrid should switch over to stand-alone mode when there is a case of power fail in the main grid or during a period excess power generation. The proposed DVR operating in microsource mode, presents two advantages over previous DVR models : 1) it allows power to be exported to the priority loads and 2) it allows excess energy to be stored. Therefore, the proposed DVR has higher utilization rate than conventional one in distribution networks. The operational flowchart of EMS in this mode is shown in Fig. 2 (c).

\section{The Proposed Composite Controller}

In fact, microgrids are small-scale, low-voltage supply networks designed to supply electrical and heat loads [18]. Inconsideration of a cost effective means to improving the efficiency of the proposed DVR system, a transformless structure is used in the proposed DVR system and this structure could avoid the harmonic pollution caused by series transformer. However, the microsources in Microgrids must be equipped with power electronic interfaces (PEIs) and controls to provide the required flexibility and reliable power, the abundant use of PEIs also cause much harmonic to the power supply. In this paper, a composite controller for the proposed DVR system in the

Microgrid is presented, which could ensure the compensation ability of voltage sag or other disturbance and compensate harmonic voltage in the specified order by DVR. The complete block diagram of the proposed composite control strategy is shown in Fig. 3.

In Fig. 3, $U_{S}$ is distorted voltage and $U_{L}$ is undistorted voltage, $U_{D V R}$ is the actual output voltage of the DVR and voltage $U^{*}{ }_{D V R}$ is the reference voltage. The cascaded H-bridge MLI is series connected with the system using a LC filter $\left(L_{f}\right.$ and $\left.C_{f}\right)$. The $d q$ detection algorithm and pre-compensation methods have been used in the voltage sag detection part and the reference voltage generated part, respectively.

\subsection{Analysis and design of parameters}

The introduction of proposed Modified ProportionalResonant (MPR) controller is based on the conditions that the bandwidth of conventional PR controller is relatively narrow and the characteristic of anti-frequency offset is not so satisfied [19]. The transfer function of MPR controller is expressed as (1).

$$
G_{M P R}(s)=K_{p}+\sum_{k=3,5,7 \ldots} \frac{2 K_{r} \omega_{c} s}{s^{2}+2 \omega_{c} s+\left(k \omega_{0}\right)^{2}}
$$

where $K_{p}$ and $K_{r}$ are proportional and resonant gain, respectively, $\omega_{c}$ is cut-off frequency and $\omega_{0}$ is resonant frequency, $k$ is harmonic in specified order.

When $K_{p}=10, K_{r}=15, \omega_{c}=25 \mathrm{rad} / \mathrm{s}, \omega_{0}=100 \pi \mathrm{rad} / \mathrm{s}$, the bode diagrams of MPR controller and PR controller are illustrated in Fig. 4 (a). The comparative analysis of amplitude-frequency curves in Fig. 4 (a) shows that MPR controller has a much wider bandwidth than the PR controller, which means that the MPR controller could produce a large amplitude gain when the system frequency 


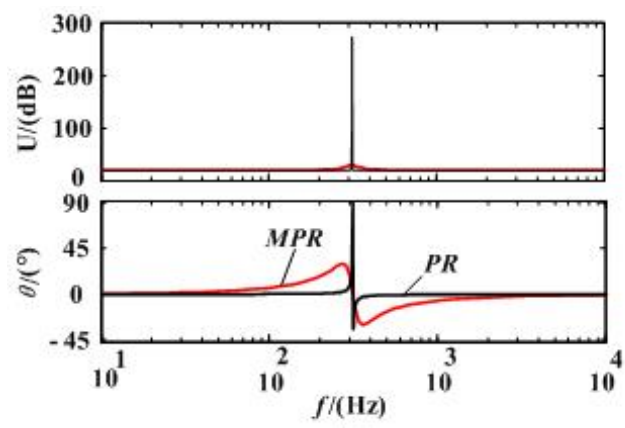

(a)

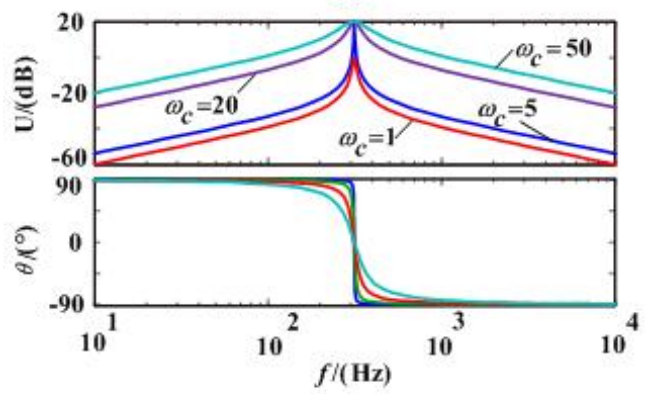

(c)

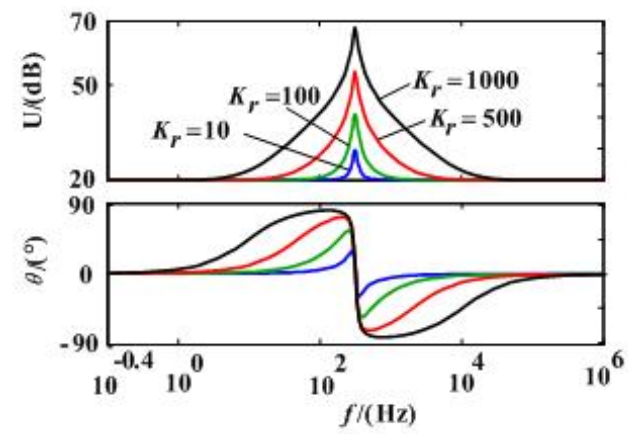

(b)

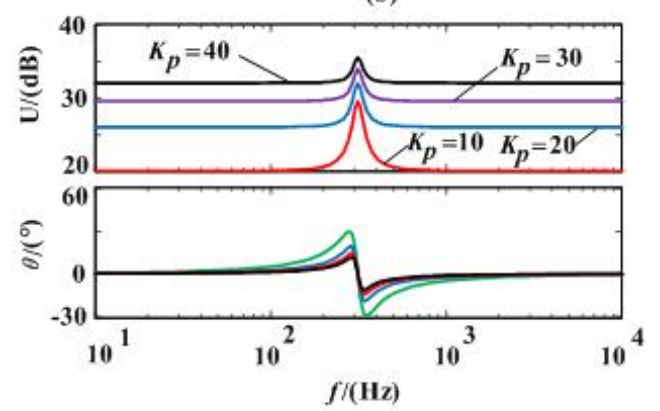

(d)

Fig. 4. The parameters analysis of MPR controller using bode diagram: (a) is the bode diagram of MPR controller and PR controller; (b) is the bode diagram of MPR controller in case of varying $K_{\mathrm{r}}$; (c) is the bode diagram of MPR controller in case of varying $\omega_{\mathrm{c}} ;(\mathrm{d})$ is the bode diagram of MPR controller in case of varying $K_{\mathrm{p}}$

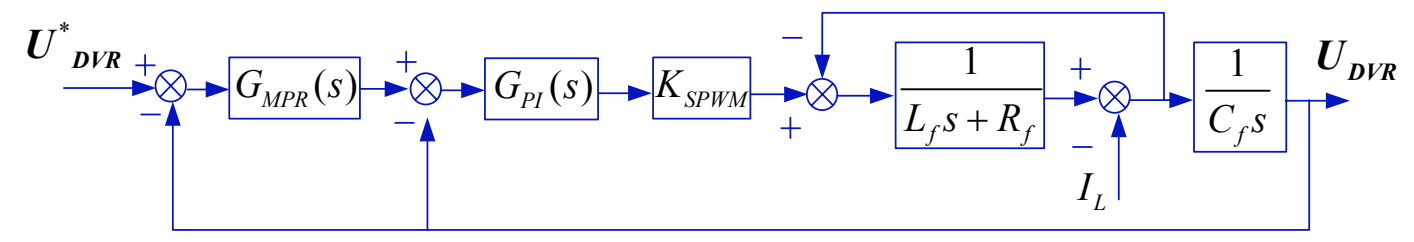

Fig. 5. The equivalent block diagram of proposed composite control strategy

$$
\begin{aligned}
& U_{D V R}=\frac{G_{M P R}(s) K_{S P W M} G_{P I}(s)}{L_{f} C_{f} s^{2}+R_{f} C_{f} s+s C_{f} K_{S P W M} G_{P I}(s)+G_{M P R}(s) K_{S P W M} G_{P I}(s)+1} U^{*}{ }_{D V R} \\
& -\frac{L_{f} s+R_{f}}{L_{f} C_{f} s^{2}+R_{f} C_{f} s+s C_{f} K_{S P W M} G_{P I}(s)+G_{M P R}(s) K_{S P W M} G_{P I}(s)+1} I_{L} \\
& G_{P I}(s)=K_{P}+\frac{K_{I}}{s} \quad, \quad G_{M P R}(s)=K_{p}+\sum_{k=3,5,7 \ldots} \frac{2 K_{r} \omega_{c} s}{s^{2}+2 \omega_{c} s+\left(k \omega_{0}\right)^{2}}
\end{aligned}
$$

offset occurs. As (1) shows, the factor influencing the performance of the MPR controller is not unique. To obtain better dynamic and static performance, the rational design of the controller parameters is especially significant. To analyze the effect of every single parameter on the MPR controller, two of the three parameters were kept constant and the third one was altered. The influence of each parameter to the amplitude-frequency characteristic of MPR controller is demonstrated in Fig. 4 (b), (c) and (d), respectively. As is shown in Fig. 4 (b), the magnitude of MPR controller increases with the increase of $K_{p}$. As a result, the parameter $K_{p}$ mainly affects the proportional gain of MPR controller. In Fig. 4 (c), when $K_{r}$ increases, the amplitude-frequency curve shifts upward. The magnitude of MPR controller at resonant frequency also increases, while the bandwidth remains the same. Therefore, $K_{r}$ plays a role in reducing steady-state error. As is shown in Fig. 4 (d), when $\omega_{c}$ changes, the magnitude at resonant frequency remains relatively without change. Whereas the magnitudes and bandwidths at other frequencies increase with $\omega_{c}$. Thus, the bandwidth of the improved PR controller is mainly affected by $\omega_{c}$. 


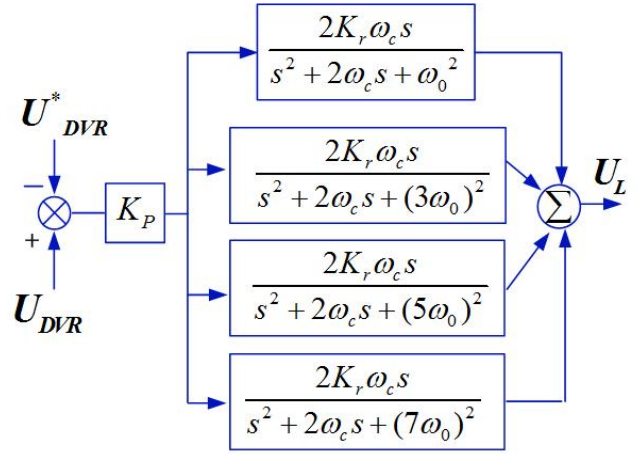

(a)

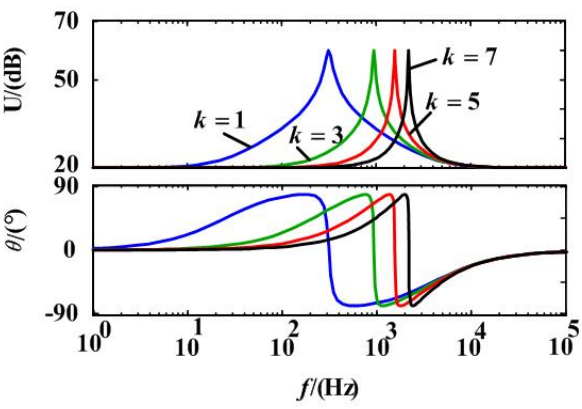

(b)

Fig. 6. The Structure and bode analysis of MPR controller with harmonic compensation: (a) is the structure diagram and (b) is the bode diagram

\subsection{Analysis of compensation performance}

To facilitate the analysis, Fig. 3 is converted equivalently into Fig. 5. The transfer function of $U_{D V R}$ could be obtained by using Mason gain formula as (2) described, where $G_{P I}(s)$ and $G_{M P R}(s)$ are described as formula (3). Detailed derivation of the transfer function is provided in the Appendix.

Fig. 4 (a) shows the transfer function of MPR controller $G_{M P R}(s)$ could obtain infinite gain values at the frequency $\omega_{0}$, which leads to a deduction of (2) expressed in the simple expression as

$$
U_{D V R} \approx U^{*}{ }_{D V R}
$$

It is apparent by observation in (4) that the proposed composite controller could achieve zero-steady error of the compensation voltage.

\subsection{Analysis of harmonic compensation}

To avianize the influence of specified harmonic on system voltage, the function of compensation to harmonic voltage in specified order is designed in presented composite controller. The structure diagram and bode diagram of voltage-loop MPR controller with compensation to harmonic voltage in specified order are shown in Fig. 6 (a) and (b), respectively. As is illustrated in Fig. 6, it is clear to see that since the resonance response is produced only in the designated resonant-frequency point, and the resonance response of specified harmonic do not interfere with each other.

\section{Simulation results}

As a first approach to verify the performance of the proposed DVR system in the Microgrid, a simulation model is built using the Matlab/Simulink's Sim Power Systems platform. A $1 \mathrm{kVA} / 380 \mathrm{~V} / 50 \mathrm{~Hz}$ system is selected
Table 2. Simulation parameters

\begin{tabular}{c|c|c}
\hline Description & Parameter & Value \\
\hline \multirow{2}{*}{ AC supply } & Nominal voltage & $380 \mathrm{~V}$ \\
& System frequency & $50 \mathrm{~Hz}$ \\
\hline \multirow{2}{*}{ Transformer } & Capacity & $2.5 \mathrm{MVA}$ \\
& Voltage rating & $380 / 220 \mathrm{~V}$ \\
\hline Wind turbines & Maximum power & $0.8 \mathrm{MW}$ \\
\hline \multirow{2}{*}{ Battery bank } & Nominal voltage & $24 \mathrm{~V}$ \\
& Capacity & $800 \mathrm{Ah}$ \\
\hline \multirow{2}{*}{ Priority load } & Resistance & $20 \Omega$ \\
& Inductance & $100 \mathrm{mH}$ \\
\hline \multirow{2}{*}{ Casecaded H-bridge } & DC bus & $520 \mathrm{~V}$ \\
MLI & DC capacitance & $2000 \mathrm{uF}$ \\
& Filter inductance & $100 \mathrm{mH}$ \\
& Filter capacitance & $4.7 \mu \mathrm{f}$ \\
MPR controller & Switching frequency & $5 \mathrm{kHz}$ \\
\hline \multirow{2}{*}{ MP } & $K_{p}, K_{r}, \omega_{c}$ & $10,500,20 \mathrm{rad} / \mathrm{s}$ \\
\hline
\end{tabular}

for the simulation and complete list of numeric parameters is presented in Table 2. Simulation is carried out in accordance with the above three modes and the results of analysis are as follows.

\subsection{Compensation mode}

An illustrative set of waveforms is present in Fig. 7, and the DVR is turned-on at $\mathrm{t}=0.07 \mathrm{~s}$. As shown in Fig. 7 (a), with the system operating in the steady state, a five-cycle sag of 0.2 p.u. occurs in all the phases of supply voltage at $\mathrm{t}=0.07 \mathrm{~s}$. The sag is cleared at $\mathrm{t}=0.14 \mathrm{~s}$. The detection voltage and actual output voltage of DVR are shown in Fig. 7 (b) and (c), respectively. Fig. 7 (d) shows the undistorted voltage of critical load, it is obvious that the load-side voltage is effectively regulated to its nominal value. When the sag occurs, the load voltage is recovered to 1 p.u. even during the transition states at the beginning and ending instants of the sag. The results reflect the accurate performance of the proposed DVR system in the Microgrid.

Fig. 8 shows the performance of harmonics compensation of the proposed controller compared with other existing controller of DVR. The line-to-neutral voltage at 

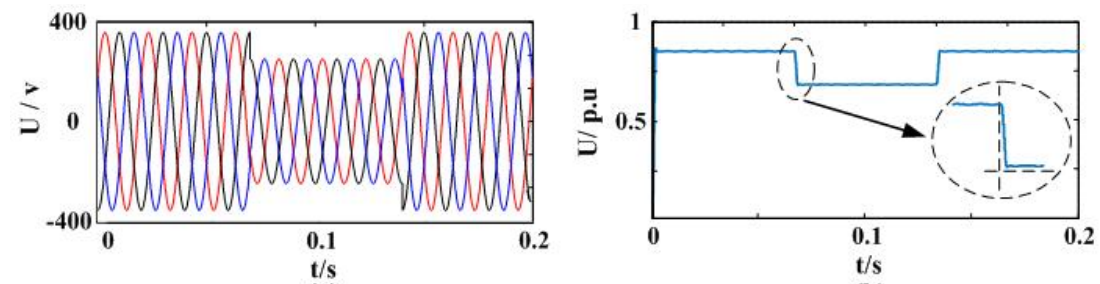

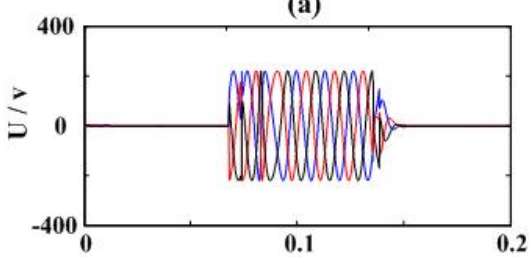

(c)

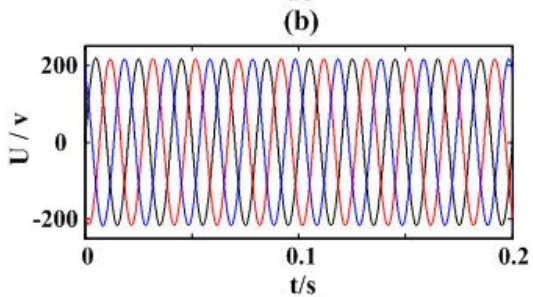

(d)

Fig. 7. The simulation results: (a) is the distorted supply-side voltage; (b) and (c) are the detection voltage and actual output voltage of proposed DVR, respectively; (d) is the undistorted load-side voltage.

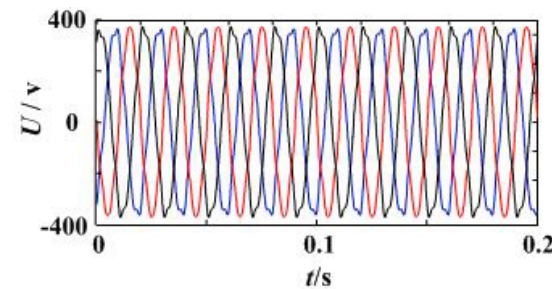

(a)

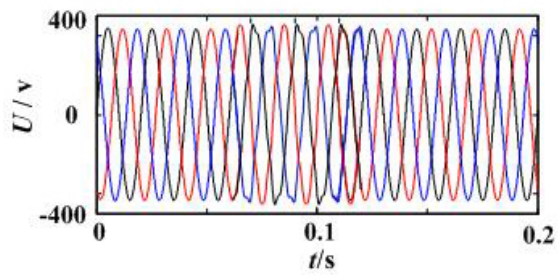

(c)

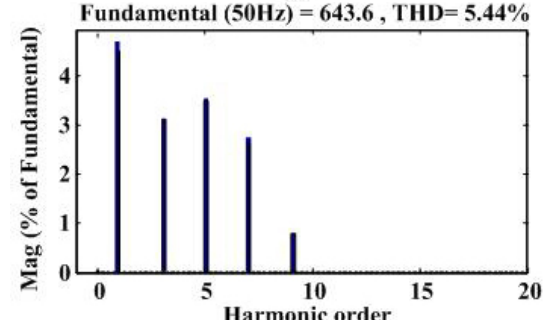

(e)

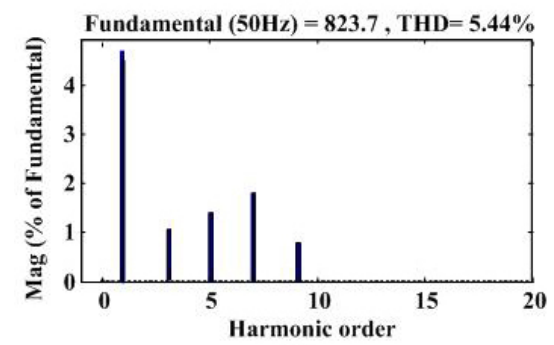

(g)

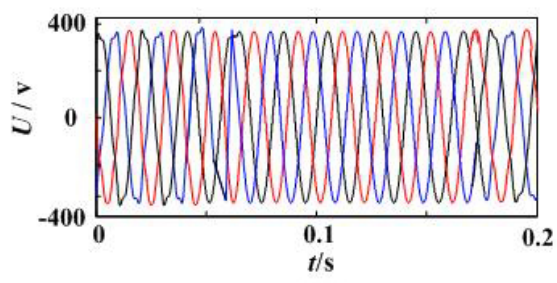

(b)

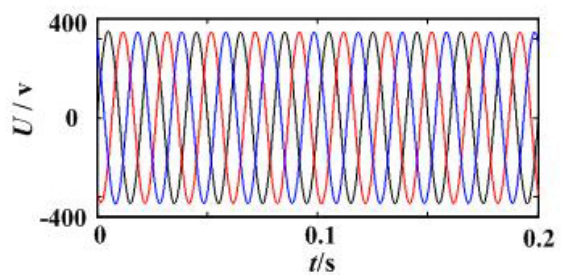

(d)

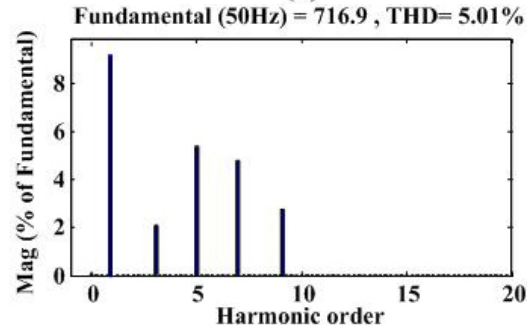

(f)

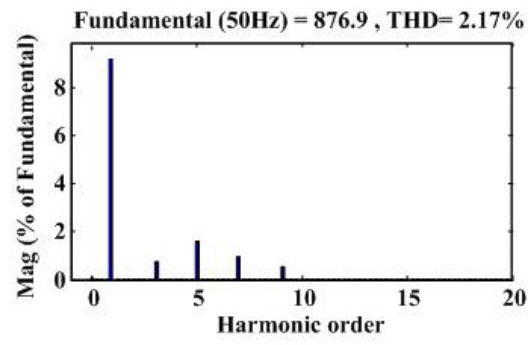

(h)

Fig. 8. The simulation results. (a) is the supply-side voltage without DVR, (b) and (c) are the supply-side voltage with DVR using existing open-loop controller and multi-loop controller, respectively, and (d) is the supply-side voltage with DVR using the proposed controller. (e) is the THD analysis of supply-side voltage without DVR, (f) and (g) are the THD analysis of supply-side voltage with DVR using existing open-loop controller and multi-loop controller, respectively, and (h) is the THD analysis of supply-side voltage using the proposed controller 


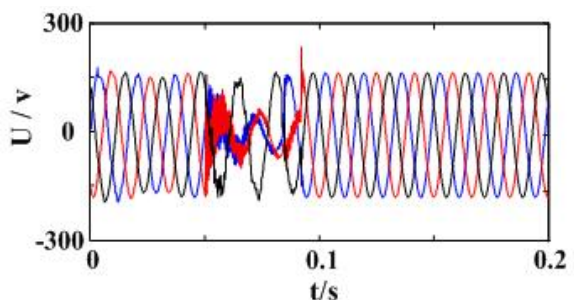

(a)

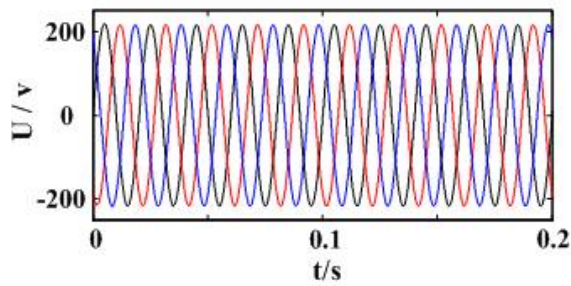

(c)

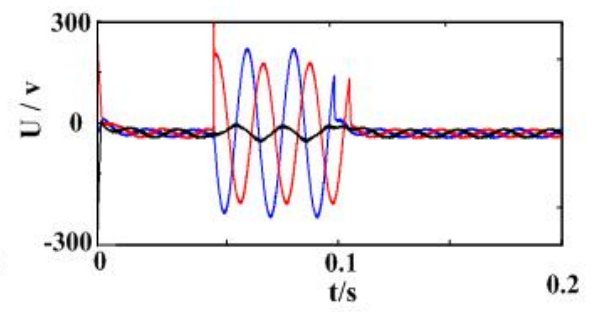

(b)

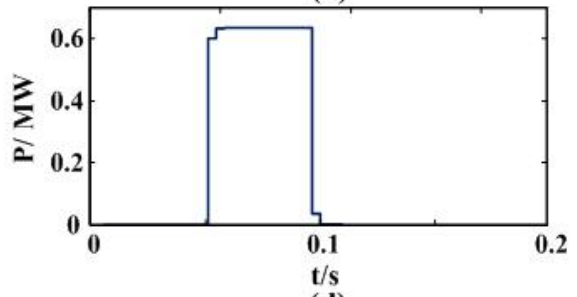

(d)

Fig. 9. The simulation results: (a) is the load-side voltage without DVR; (b) is the output voltage of proposed DVR; (c) is the load-side voltage with DVR and (d) is the output active power of batteries.

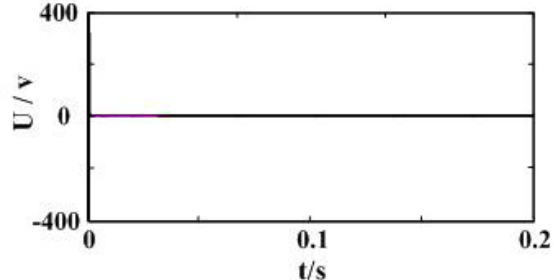

(a)

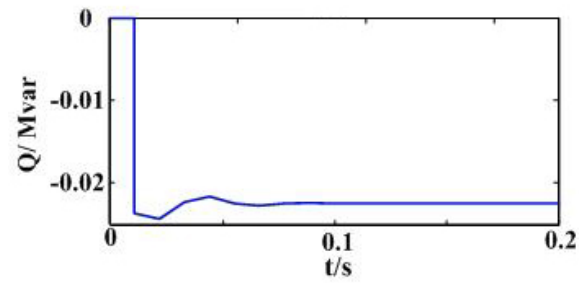

(c)

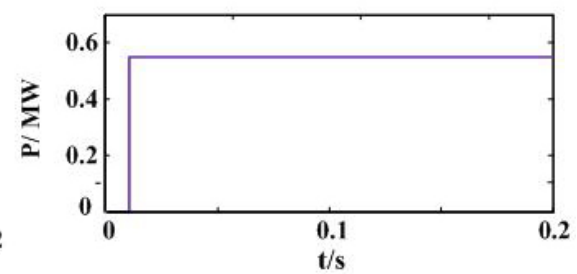

(b)

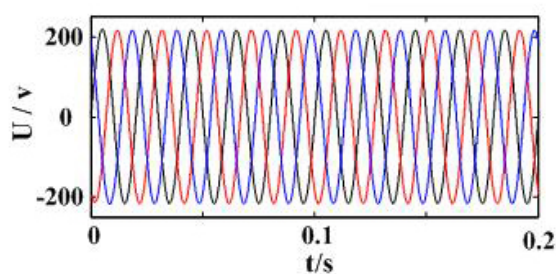

(d)

Fig. 10. The simulation results: (a) is the supply-side voltage; (b) and (c) are the output active power and reactive power of wind turbines, respectively and (d) is the load-side voltage with proposed DVR.

the PCC is illustrated in Fig. 8 (a) when the main grid voltage is polluted with harmonics. The results of the FFT applied to those waveforms are depicted in Fig. 8 (e) for key harmonics. The THD of supply-side is $5.44 \%$, which clearly exceed the IEC limits [20]. Fig. 8 (b) and (c) illustrate the supply-side voltage waveforms by using existing open-loop and multi-loop controller, respectively. Their FFT analysis could be seen in Fig.8 (f) and (g), respective [21]. The observation can be clearly seen that open-loop controller could hardly restrain the harmonic component effectively. When restraining the harmonic component, the proposed controller in [21] called multiloop controller needs wider bandwidth of the current loop controller and faster response speed, which limits its ability to compensate the harmonic voltage [22]. Note that those two existing controller all hold the voltage compensation ability. Fig. 8 (d) and (h) are the supply-side voltage waveforms with proposed DVR and its FFT analysis. Clearly, using the proposed DVR model, the THD is drastically reduced to $2.17 \%$ and conforms to the IEC limits.

\subsection{UPS mode}

The Microgrid remains connected to the main grid at the beginning state, and it switches over to stand-alone mode at $\mathrm{t}=0.05 \mathrm{~s}$, this period lasts $0.05 \mathrm{~s}$. Fig. 9 (a) and (b) show the load-side voltage in the Microgrid without and with DVR, respectively. By contrast, it is obvious that the proposed DVR can operate in the UPS mode, eliminate 
negative effects to the load-side voltage effectively and avoid the economic losses caused by the operation modes switching of the Microgrid. Fig. 9 (c) is the output active power of batteries in this mode.

\subsection{Microsource mode}

When the Microgrid operates in stand-alone mode, the proposed DVR system could act as the role of power supply. It could export the power to those priority loads during excess power generation on wind turbines. In this case, the supply-side voltage is shown in Fig. 10 (a). Fig. 10 (b) and (c) show the output active power and reactive power of wind turbines, respectively, and (d) is the loadside voltage with the proposed DVR. This could reduce the power supply from main grid and be favorable for energy saving and emission reduction.

\section{Conclusion}

To dissatisfied voltage quality of the Microgrid, a DVR model based on wind-turbine generation and battery units is proposed in this paper. Three operating modes of the pro-posed DVR, namely the compensation mode, the UPS mode and the micro-source mode, are put forward. Compared to other conventional types of DVR models in distribution networks, the proposed DVR has higher utilization rate. The proposed DVR system is based on a conventional cascaded H-bridge MLI topology, and a new composite control strategy composed of double closed-loop PI controller and Modified MPR controller is presented to make the DVR enable to further reduce voltage harmonic characteristics of the system when making compensation of load voltage. The theoretical analysis and simulation results all validate the robustness and accuracy of the proposed DVR model.

\section{Acknowledgements}

This work is supported by A Project Funded by the Priority Academic Program Development of Jiangsu Higher Education Institutions (PAPD) and Jiangsu province Natural Science Foundation(BE2010354).

\section{References}

[1] Lu Z. X, Wang C. X, Min Y," Overview on microgrid research," Automation of Electric Power System, vol. 31, no. 19, pp. 100-107, Oct.2007.

[2] Il-Yop C, Wenxin L, Cartes D A, "Control methods of inverter-interfaced distributed generators in a microgrid system," IEEE Transaction on Industrial
Applications, vol. 46, no.3, pp. 1078-1088, Jun. 2010.

[3] Cheng R. F, Han X., "Research on operation modes of dynamic voltage restorer in microgrid," Power System Technology, vol. 37, no. 3, pp. 610-615, Mar. 2013.

[4] P. C. Loh, D. Li, Y. K. Chai, F. Blaabjerg, "Autonomous operation of hybrid microgrid with $\mathrm{AC}$ and DC subgrids," IEEE Transaction on Power Electronics, vol. 28, no. 5, pp. 2214-2223, May 2013.

[5] Irena Wasiak, Ryszard Pawelek, Rozmyslaw Mienski, "Energy storage application in low-voltage microgrids for energy management and power quality improvement," IET Generation, Transmission \& Distribution, vol. 8, pp. 463-472, Mar.2014.

[6] Babaei, E., Farhadi Kangarlu, M., Sabahi, M., "Compensation of voltage disturbances in distribution systems using single-phase dynamic voltage restorer," Electric Power System Research, vol. 80, no.12, pp. 1413-1420, Dec.2010.

[7] Rosas-Caro, J. C. , etc., "Two-switch three-phase aclink dynamic voltage restorer," IET Power Electronic $s$, vol. 5, no.9, pp. 1754-1763,Nov. 2012.

[8] Massoud, A. M., Ahmed, S., Enjeti, P. N., Williams, B. W., "Evaluation of a multilevel cascaded-type dynamic voltage restorer employing discontinuous space vector modulation," IEEE Transaction on Industrial Electronic, vol. 57, no. 7, pp. 2398-2410, Jul. 2010.

[9] M. Ramasamy, S. Thangaval, "Experimental verifycation of PV based Dynamic Voltage Restorer (PVDVR) with significant energy conservation," Electrical Power and Energy Systems, vol. 49, no. 1, pp. 296307, Jul. 2013.

[10] Ebrahim Babaei, Mohammad Farhadi Kangarlu, Mehran Sabahi, "Dynamic voltage restorer based on multilevel inverter with adjustable dc-link voltage," IET Power Electronics, vol. 7, no. 3, pp. 576-590, M ar. 2014.

[11] Visser, A. J., Enslin, J. H. R., Mouton, H. T., "Transformerless series sag compensation with a cascade multilevel inverter," IEEE Transaction Industrial Electronic, vol. 49, no. 4, pp. 824-831, Aug. 2002.

[12] Loh, P. C., Vilathgamuwa, D. M., Tang, S. K., Long, H. L., "Multilevel dynamic voltage restorer," IEEE Power Electronic Letter, vol. 2, no. 4, 2005, pp. 125130.

[13] Sasitharan, S., Mishra, M. K., "Constant switching frequency band controller for dynamic voltage restorer," IET Power Electronics, vol. 3, no. 5, pp. 657-667, Sept. 2010.

[14] G. J. Li, X. P. Zhang, S. S. Choi, T. T. Lie and Y. Z. Sun, "Control strategy for dynamic voltage restorers to achieve minimum power injection without introducing sudden phase shift," IET Generation, Transmission \& Distribution, vol. 1, no. 5, pp. 847-853, 
Sept. 2007.

[15] Huang Yonghong, Xu Junjun, et al, "Dynamic Voltage Restorer in Micro-grid Based on a Minimum Energy Compensation Control Scheme," Transaction of China Electrotechnical Society, vol. 29, no.12, pp. 128-136, Dec. 2014.

[16] Wang, B., Venkataramanan, G., Illindala, M., “Operation and control of a dynamic voltage restorer using transformer coupled H-bridge converters," IEEE Transaction on Power Electronic, vol. 21, no. 4, pp. 1053-1061, Jul. 2006.

[17] Roncero-Sanchez, P., Acha, E., "Dynamic voltage restorer based on flying capacitor multilevel converters operated by repetitive control," IEEE Transaction Power Deliver, vol. 24, no. 2, pp. 951960, Apr. 2009.

[18] S. Chowdhury, S. P. Chowdhury and P. Crossley, "Microgrids and Active Distribution Networks," IET Renewable Energy Series 6, London, United Kingdom, 2009.

[19] Chen S, Lai Y M, Tan S C, "Analysis and design of repetitive controller for harmonic elimination in PWM voltage source inverter systems," IET Power Electronics, vol. 1, no. 4, pp. 497-506, Dec. 2008.

[20] IEC 61000-3-6. Technical report. Limits assessment of emission limits of the connection of distorting installations to MV. HV and EHV; 2008.

[21] Mahinda V., A. A. D. Ranjith Perera, and S. S. Choi, "Performance Improvement of the Dynamic Voltage Restorer With Closed-Loop Load Voltage and CurrentMode Control," IEEE Transaction on Power Electronic, vol. 17, no. 5, pp. 824-834, Sept.2002.

[22] Shi Y., Yang H., "A Control Strategy for Dynamic Voltage Restorer With Harmonic Compensation Function," Electrical Power Automation Equipment, vol. 26, no. 5, pp. 88-91, May, 2006.

\section{Appendix}

When only considering $U^{*}{ }_{D V R}$ acting on the entire system, there is a single forward path and three separate closed-loop paths:

$$
p_{1}=\frac{G_{M P R}(s) G_{P I}(s) K_{S P W M}}{L_{f} C_{f} s^{2}+R_{f} C_{f} s}, \Delta_{1}=1
$$

In this case, using Mason gain formula analysis, the transfer function of the control system can be written as

$$
\begin{aligned}
\frac{U_{D V R}}{U^{*}{ }_{D V R}} & =\frac{p_{1} \Delta_{1}}{1-\left(L_{1}+L_{2}+L_{3}\right)}=\frac{G_{M P R}(s) K_{S P W M} G_{P I}(s)}{H(s)} \\
H(s) & =L_{f} C_{f} s^{2}+R_{f} C_{f} s+s C_{f} K_{S P W M} G_{P I}(s) \\
& +G_{M P R}(s) K_{S P W M} G_{P I}(s)+1
\end{aligned}
$$

When only considering $I_{L}$ acting on the entire system, there is a single forward path and a single closed-loop path:

$$
\begin{gathered}
p_{1}=-\frac{1}{C_{f} s}, \Delta_{1}=1 \\
L_{1}=\frac{G_{P I}(s) K_{S P W M}}{L_{f} C_{f} s^{2}+R_{f} C_{f} s}
\end{gathered}
$$

In this case, using Mason gain formula analysis, the transfer function of the control system can be written as

$$
\begin{aligned}
& \frac{U_{D V R}}{I_{L}}=\frac{p_{1} \Delta_{1}}{1-L_{1}}=-\frac{L_{f} s+R_{f}}{H(s)} \\
H(s)= & L_{f} C_{f} s^{2}+R_{f} C_{f} s+s C_{f} K_{S P W M} G_{P I}(s) \\
+ & G_{M P R}(s) K_{S P W M} G_{P I}(s)+1
\end{aligned}
$$

Therefore, the transfer function of the entire system can be expressed as

$$
\begin{gathered}
U_{D V R}=\frac{G_{M P R}(s) K_{S P W M} G_{P I}(s)}{H(s)} U^{*}{ }_{D V R}-\frac{L_{f} s+R_{f}}{H(s)} I_{L} \\
H(s)=L_{f} C_{f} s^{2}+R_{f} C_{f} s+s C_{f} K_{S P W M} G_{P I}(s) \\
+G_{M P R}(s) K_{S P W M} G_{P I}(s)+1
\end{gathered}
$$

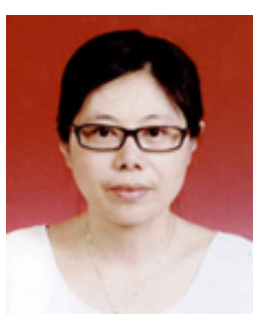

Huang Yonghong She received the M.Sc. degree and the Ph.D. degree in control theory and engineering from Jiangsu University, Zhenjiang, China, in 1998 and 2009 respectively.

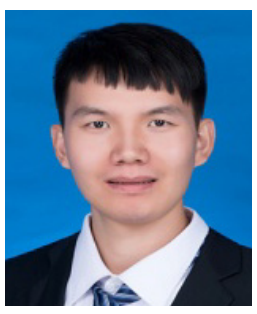

Xu Junjun He received the M.Sc. degree in school of electrical and information engineering from Jiangsu University, Zhenjiang, China, in 2015. Now he is pursuing his $\mathrm{Ph}$. D. degree in school of electrical engineering from Southeast University, Nanjing, China.

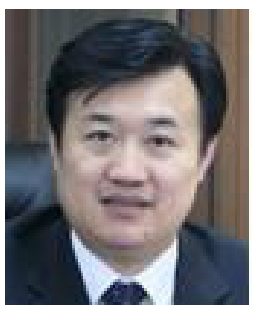

Sun Yukun received the B.Sc degree in control theory and engineering from Jiangsu University, Zhenjiang, China, in 1982 and the Ph.D. degree in Mechanical Manufacture and Automation from Jiangsu University, Zhenjiang, China, in 2006. Since 1982, he has been with Jiangsu University, where he 
is currently a professor in the School of Electrical Information Engineering. he research interests include magnetic levitation motor and its control and power quality control.

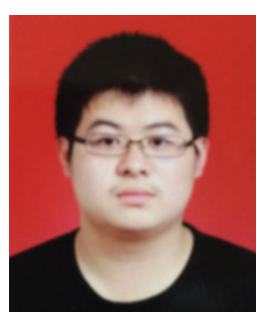

Huang Yuxiang is currently working toward the B.Sc degree electrical engineering at Jiangsu University, Zhenjiang, China. His research interests include motor drives for electric vehicles and power quality control. 